\title{
The Effects of Different Plant Activators on Protein, Lipid and Fatty Acids in Snack-Seed Pumpkin
}

\author{
Aygül DAYAN ${ }^{1, a^{*}}$, Nebahat SARI ${ }^{2, b}$, Fatih OZOGUL $L^{3, c}$ \\ ${ }^{1}$ Department of Plant and Animal Production, Cukurova University, Adana, Turkey \\ ${ }^{2}$ Department of Horticulture, Cukurova University, Adana, Turkey \\ ${ }^{3}$ Department of Seafood Processing Technology, Cukurova University, 01330 Adana, Turkey \\ a email: adayan@cu.edu.tr, bemail: nesari@cu.edu.tr, ${ }^{c}$ email: fozogul@cu.edu.tr
}

Keywords: Snack pumpkin, plant activators, chemical analysis

\begin{abstract}
In this study, Nusem and Beppo snack seed pumpkin cultivars were used to determine the effects of different plant activators on seed protein, lipid and fatty acids contents. In the context of study, plant activators consist of Crop-set (CR), EM1, ERS, Vitormone-Plus Drip (VIT), Bacillus subtilis (OSU 142), Bacillus megatorium (M3), Azospirillum sp. (SP 245), Spirulina platensis (SIP), Ecocompost (EKO), Camli Botanica liquid organic fertilizer (BOT) and Zincon (ZIN) were used as organic fertilizer. In the experiment, the plant activators were applied to the plants alone or in combination with each other and organic fertilizer. Two separate control groups which were organic and conventional (CONV.) fertilizer have been identified. As a result of the use of different plant activators, the highest protein content was obtained from CONV. application (35.50\%), M3+SP 245 (33.09\%) and M3 (33.04\%); the highest lipid content was observed from SP 245+OG (45.90\%), CR $(44.48 \%)$ and SIP+OG (44.26\%) applications. The use of different plant activators effected the fatty acid contents of seeds. Total 11 fatty acids were identified. Among the fatty acids, C16:0 (Palmitic acid), C18:0 (Stearic acid), C18:1 (Oleic acid) and C18:2 (Linoleic acid) were found dominant.
\end{abstract}

\section{Introduction}

Snack pumpkin product is the seed of plant from Cucurbita pepo and Cucurbita moschata. Seeds not only contain snack food but are also sources for food raw materials. Turkey produces about 42181 tons Snack pumpkin in 628441 area [1]. Pumpkin seed (Cucurbita pepo L.) is high in oil, protein, and total unsaturated fatty acids and provides an important source of nutrition [2]. Snack pumpkin showed rice produce both area and quantity. But, despite the increase in production quantities, the amount of product received from the unit area did not reach adequate levels. For this purpose the increase yield and quality used microbial fertilizer in addition plant activators [3]. Plant activators contains alone such as Plant Growth Promoting Rhizobacteria= PGPR; Azotobacter, Azospirillum and Bacillus subtilis, Bacillus firmus, Pseudomonas striata etc.), mycorrhiza (Glomus fasciculatum, Glomus intraradices, Glomus aggregatum etc.), microalgae (Spirulina platensis) microorganism or combination these microorganism with mineral element, vitamin, plant extract etc. The present study was carried out to examine the effect of different plant activators on total proteins, total lipid, fatty acids of snack-seed pumpkin.

\section{Materials and Methods}

This study was conducted at the experimental field in Cukurova University Pozanti Agricultural Research and Application Center. "Nusem" snack seed pumpkin and "Beppo" naked seed pumpkin were used as plant material. These snack pumpkins were cut and seeds extracted in 07 July 2015 and 21 July 2016 respectively, the seeds dried about ten days for analysis. 5-10 g powdered seed sample was taken for determination of lipid and $1 \mathrm{~g}$ for protein analysis.

Plant activators were Crop-set (CR), EM1, ERS, Vitormone-Plus Drip (VIT), Bacillus subtilis (OSU 142), Bacillus megatorium (M3), Azospirillum sp. (SP 245), Spirulina platensis (SIP). CR, contains crop fermentation of Lactobacillus acidiophilus and plant extract, manganese, copper, iron, 
magnesium, zinc. CR was applied $60 \mathrm{ml}$ in ha area. EM1, contains photosynthesis bacteria, lactic acid bacteria, yeast, actinomycetes and molds. The application was made once every two weeks. EM1; mixed 11 EM1 with 181 water and then completed to 201.41 were taken from this mixture. ERS, Glomus intraradices (25\%), G. mosseae (24\%), G. aggregatum (1\%), G. monosporum (1\%), G. deseticola (1\%), G. brasilianum (1\%), G. etunicatum (1\%), G. margarita (1\%) are mycorrhiza fungi. The seedling has been dipped before planting. Application dose $250 \mathrm{~g} / \mathrm{da}$. VIT (Azotobacter chroococum and Azotobacter vinelandi) once application, the seedling has been dipped before planting. The second application was made 2 months after the planting. Application dose $200 \mathrm{cc} / \mathrm{da}$. Bacillus subtilis (OSU 142), Bacillus megatorium (M3), Azospirillum spp. (SP 245) were obtained from Yeditepe University, Turkey. 1/10 mixture was prepared from the bacterial isolate and taken at

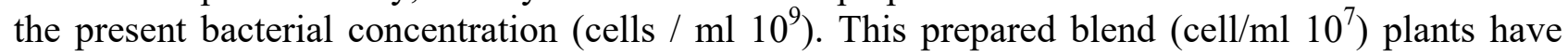
been dipped for 20 minutes before planting and then planted. Spirulina platensis is among the oldest and primitive life forms on earth and comes from the phylum of very basic life forms, cyanobacteria (better known as blue-green algae) and naturally contain various plant nutrients. $45 \mathrm{~g} / \mathrm{l}$ Spirulina spp. solution has been prepared, and $200 \mathrm{cc}$ of this solution has been applied to the plants, near the seedling roots. Ecocompost (EKO), Camli Botanica liquid organic fertilizer (BOT) and Zincon (ZIN) were used as organic fertilizer. Ecocompost is a fertilizer $100 \%$ organic, contains $\mathrm{N}, \mathrm{P}, \mathrm{K}, \mathrm{Ca}, \mathrm{S}, \mathrm{Mg}, \mathrm{Fe}, \mathrm{Mn}, \mathrm{Zn}$ and applied before planting per plant $250 \mathrm{~g}$ and after 1 month planting $200 \mathrm{~g}$ per plant. Camlı Botanica Liquid organic fertilizer contains humic and fulvic asit $(24 \%)$, organic matter $(35 \%)$, nitrogen $(\mathrm{N}, 4 \%)$, organic nitrogen $(\mathrm{N}, 3.2 \%), \mathrm{P}_{2} \mathrm{O}_{5}(2 \%), \mathrm{K}_{2} 0(3.5 \%)$ and applied after a week seedling planting, lastly 1 month after from this apply. Zincon, Zn (11\%), (Mn 2\%) and B (2.5\%), Acetylsalicylic acid (0.01\%), Free amino acid (5\%), organic acid (11\%), organic carbon of biological origin (10\%), B1-B2-B6-B12 (6\%) vitamin, vitamin, phytohormone, enzym, humic acid. Apply when planted seedling after 15 day, $750 \mathrm{cc}-1 \mathrm{l} / \mathrm{da}$ and second application was given at $750 \mathrm{cc}-11 /$ ha when the first fruits were observed. Conventionally fertilizer have been used $100 \mathrm{~kg} \mathrm{~N}: 50 \mathrm{~kg} \mathrm{P}_{2} \mathrm{O}_{5}: 200 \mathrm{~kg} \mathrm{~K} / 2 \mathrm{O} / \mathrm{ha}$.

As organic fertilizers (OG); Ecocompost, Camlı Botanica Liquid organic fertilizer and Zincon have been used. In the experiment, the plant activators were applied to the plants alone or in combination with each other and organic fertilizer. Two separate control groups which were organic and conventional (CONV.) fertilizer have been identified. The following methods were used: Kjeldahl method [4] for total protein, and Bligh and Dyer method [5] was used for total oil; powdered seed samples were measured in quantities ranging from 5-10 $\mathrm{g}$ and placed in to the cellulose cartridges. Over the cartridge was pressed well with cotton on the purpose of that the samples should not diffuse. The cellulose cartridge was placed on a glass ballon and extractioned with diethyl ether solution for 3 hours. Diethyl ether was evaporated from the diethyl ether-lipid fraction which have been remained in the glass balloons using the method of a rotary evaporator in a water bath at $65^{\circ} \mathrm{C}$. After that, the balloons were waited at stand at $60^{\circ} \mathrm{C}$ for 20 minutes in the stove to evaporate of the diethyl ether, they cooled until the room temperature in a desiccator and weighed on a $0.1 \mathrm{mg}$ sensitive precision scale. The following formula has been used in the calculation of the lipid ratio Kenar [6].

Lipid ratio $(\%)=[$ Glass balloon weight $(\mathrm{g})+$ Lipid $(\mathrm{g})]$-[Glass balloon $(\mathrm{g})]$ x100/ Sample weight $(\mathrm{g})$

Fatty acid profiles of fat extracted from the seed snack pumpkin samples were determined according to the method described by Ichibara et al. [7] with minor modification by using by gas chromatography (GC).

Statistical analyses were performed by the use of the JMP 5.1 program and Tukey test was used to compare the averages. 


\section{Results and Discussion}

At in the percent of protein, lipid and fatty acid, significant different between applications average were recorded (Table 1, 2 and 3). The protein content ranged from $28.32 \%$ to $35.50 \%$. The highest value was obtained from application of CONV. whereas applications ERS $+\mathrm{OG}$ and VIT+OG had the lowest protein content (28.32\%) (Table 1).

Table 1. Total Protein Levels Obtained from Nusem and Beppo Seeds of Different Plant Activators and Organic Fertilizer Applications (\%)

\begin{tabular}{|c|c|c|c|c|c|c|c|}
\hline \multirow[b]{2}{*}{ Applications } & \multicolumn{2}{|c|}{2015} & \multirow{2}{*}{$\begin{array}{c}\text { Applications } \\
\text { x year }\end{array}$} & \multicolumn{2}{|c|}{2016} & \multirow{2}{*}{$\begin{array}{c}\text { Applications } \\
\text { x year }\end{array}$} & \multirow{2}{*}{$\begin{array}{c}\text { Applications } \\
\text { Average }\end{array}$} \\
\hline & Nusem & Beppo & & Nusem & Beppo & & \\
\hline $\mathrm{CR}$ & $30.59 \mathrm{I}-\mathrm{S}$ & $30.59 \mathrm{I}-\mathrm{S}$ & $30.59 \mathrm{~K}-\mathrm{P}$ & $29.75 \mathrm{M}-\mathrm{W}$ & $33.75 \mathrm{C}-\mathrm{H}$ & $31.75 \mathrm{H}-\mathrm{M}$ & $31.17 \mathrm{E}-\mathrm{H}$ \\
\hline EM1 & $32.41 \mathrm{D}-\mathrm{M}$ & $32.30 \mathrm{D}-\mathrm{N}$ & 32.35 G-J & $27.61 \mathrm{~W}-\mathrm{Z}$ & $32.71 \mathrm{C}-\mathrm{K}$ & $30.16 \mathrm{M}-\mathrm{R}$ & $31.26 \mathrm{EFG}$ \\
\hline ERS & 34.35 CDE & $34.35 \mathrm{CDE}$ & $34.35 \mathrm{~B}-\mathrm{E}$ & $29.33 \mathrm{O}-\mathrm{X}$ & $31.87 \mathrm{E}-\mathrm{O}$ & $30.60 \mathrm{~K}-\mathrm{P}$ & $32.48 \mathrm{BCD}$ \\
\hline VIT & $32.87 \mathrm{C}-\mathrm{K}$ & 33.12 C-J & $32.99 \mathrm{C}-\mathrm{H}$ & $28.69 \mathrm{Q}-\mathrm{X}$ & 33.12 C-J & 30.90 I-P & $31.95 \mathrm{CDE}$ \\
\hline OSU & $33.12 \mathrm{C}-\mathrm{J}$ & $33.13 \mathrm{C}-\mathrm{J}$ & $33.12 \mathrm{C}-\mathrm{H}$ & $28.36 \mathrm{R}-\mathrm{X}$ & $33.57 \mathrm{C}-\mathrm{H}$ & 30.97 I-P & 32.05 B-E \\
\hline M3 & $34.58 \mathrm{CD}$ & $34.66 \mathrm{CD}$ & $34.62 \mathrm{BCD}$ & $29.24 \mathrm{O}-\mathrm{X}$ & $33.68 \mathrm{C}-\mathrm{H}$ & $31.46 \mathrm{H}-\mathrm{O}$ & $33.04 \mathrm{BC}$ \\
\hline SP 245 & $32.45 \mathrm{D}-\mathrm{L}$ & $32.46 \mathrm{D}-\mathrm{L}$ & 32.46 F-J & $28.24 \mathrm{R}-\mathrm{Y}$ & $35.15 \mathrm{BC}$ & $31.70 \mathrm{H}-\mathrm{N}$ & $32.08 \mathrm{~B}-\mathrm{E}$ \\
\hline SIP & $30.80 \mathrm{I}-\mathrm{R}$ & $30.80 \mathrm{I}-\mathrm{R}$ & $30.80 \mathrm{~J}-\mathrm{P}$ & $27.35 \mathrm{~W}-\mathrm{Z}$ & $32.56 \mathrm{C}-\mathrm{L}$ & $29.96 \mathrm{~N}-\mathrm{R}$ & $30.38 \mathrm{GHI}$ \\
\hline EKO & 31.45 G-P & 31.45 G-P & $31.45 \mathrm{H}-\mathrm{O}$ & $24.24 \mathrm{ab}$ & $28.79 \mathrm{P}-\mathrm{X}$ & $26.51 \mathrm{UV}$ & $28.98 \mathrm{JK}$ \\
\hline BOT & $34.17 \mathrm{C}-\mathrm{F}$ & $34.17 \mathrm{C}-\mathrm{F}$ & 34.17 B-F & $27.68 \mathrm{~V}-\mathrm{Z}$ & $31.67 \mathrm{~F}-\mathrm{O}$ & 29-67 PS & $31.92 \mathrm{CDE}$ \\
\hline ZIN & $32.87 \mathrm{C}-\mathrm{K}$ & $32.87 \mathrm{C}-\mathrm{K}$ & $32.87 \mathrm{D}-\mathrm{H}$ & $26.65 \mathrm{X}-\mathrm{a}$ & $32.63 \mathrm{C}-\mathrm{K}$ & 29.64 P-S & $31.26 \mathrm{EFG}$ \\
\hline $\mathrm{CR}+\mathrm{OG}$ & $32.11 \mathrm{D}-\mathrm{N}$ & $32.11 \mathrm{D}-\mathrm{N}$ & $32.11 \mathrm{H}-\mathrm{K}$ & $25.40 \mathrm{Zab}$ & $30.61 \mathrm{I}-\mathrm{S}$ & $28.00 \mathrm{STU}$ & $30.05 \mathrm{HIJ}$ \\
\hline $\mathrm{EM} 1+\mathrm{OG}$ & $33.90 \mathrm{C}-\mathrm{G}$ & $33.90 \mathrm{C}-\mathrm{G}$ & $33.90 \mathrm{~B}-\mathrm{G}$ & $25.66 \mathrm{Y}-\mathrm{b}$ & $31.17 \mathrm{H}-\mathrm{Q}$ & $28.42 \mathrm{RST}$ & $31.16 \mathrm{E}-\mathrm{H}$ \\
\hline $\mathrm{ERS}+\mathrm{OG}$ & $30.51 \mathrm{~J}-\mathrm{T}$ & $30.51 \mathrm{~J}-\mathrm{T}$ & $30.51 \mathrm{~K}-\mathrm{Q}$ & $24.45 \mathrm{ab}$ & $27.82 \mathrm{U}-\mathrm{Z}$ & $26.13 \mathrm{VW}$ & $28.32 \mathrm{~K}$ \\
\hline $\mathrm{VIT}+\mathrm{OG}$ & $30.35 \mathrm{~K}-\mathrm{V}$ & $30.35 \mathrm{~K}-\mathrm{V}$ & $30.35 \mathrm{~L}-\mathrm{Q}$ & $23.30 \mathrm{~b}$ & $29.27 \mathrm{O}-\mathrm{X}$ & $26.28 \mathrm{UV}$ & $28.32 \mathrm{~K}$ \\
\hline $\mathrm{OSU}+\mathrm{OG}$ & $33.92 \mathrm{C}-\mathrm{G}$ & $33.92 \mathrm{C}-\mathrm{G}$ & $33.92 \mathrm{~B}-\mathrm{G}$ & $23.62 \mathrm{~b}$ & $25.36 \mathrm{Zab}$ & $24.49 \mathrm{~W}$ & $29.21 \mathrm{JK}$ \\
\hline $\mathrm{M} 3+\mathrm{OG}$ & 33.19 C-I & 33.19 C-I & $33.19 \mathrm{C}-\mathrm{H}$ & $23.97 \mathrm{ab}$ & $29.94 \mathrm{~L}-\mathrm{W}$ & 26.96 TUV & $30.07 \mathrm{HIJ}$ \\
\hline SP $245+O G$ & $30.59 \mathrm{I}-\mathrm{S}$ & $30.59 \mathrm{I}-\mathrm{S}$ & $30.59 \mathrm{~K}-\mathrm{P}$ & $37.77 \mathrm{AB}$ & $27.91 \mathrm{~T}-\mathrm{Z}$ & $32.84 \mathrm{E}-\mathrm{H}$ & $31.71 \mathrm{DEF}$ \\
\hline $\mathrm{SIP}+\mathrm{OG}$ & $32.58 \mathrm{C}-\mathrm{L}$ & $32.58 \mathrm{C}-\mathrm{L}$ & 32.58 F-I & $23.48 \mathrm{~b}$ & $29.37 \mathrm{O}-\mathrm{W}$ & $26.43 \mathrm{UV}$ & $29.50 \mathrm{IJ}$ \\
\hline OSU+M3 & $32.56 \mathrm{C}-\mathrm{L}$ & $32.56 \mathrm{C}-\mathrm{L}$ & $32.56 \mathrm{~F}-\mathrm{I}$ & $30.47 \mathrm{~J}-\mathrm{U}$ & $30.41 \mathrm{~K}-\mathrm{U}$ & $30.44 \mathrm{~K}-\mathrm{Q}$ & $31.50 \mathrm{D}-\mathrm{G}$ \\
\hline OSU+SP 245 & $29.92 \mathrm{~L}-\mathrm{W}$ & $29.66 \mathrm{~N}-\mathrm{W}$ & 29.79 O-R & 29.92 L-W & $27.62 \mathrm{~W}-\mathrm{Z}$ & $28.77 \mathrm{QRS}$ & $29.28 \mathrm{IJK}$ \\
\hline M3+SP 245 & $35.15 \mathrm{BC}$ & $35.15 \mathrm{BC}$ & $35.15 \mathrm{~B}$ & $28.03 \mathrm{~S}-\mathrm{Z}$ & $34.03 \mathrm{C}-\mathrm{G}$ & $31.03 \mathrm{I}-\mathrm{P}$ & $33.09 \mathrm{~B}$ \\
\hline $\mathrm{OG}$ & $34.70 \mathrm{CD}$ & $34.70 \mathrm{CD}$ & $34.70 \mathrm{BC}$ & $24.00 \mathrm{ab}$ & $29.71 \mathrm{~N}-\mathrm{W}$ & 26.86 TUV & $30.78 \mathrm{FGH}$ \\
\hline CONV. & $38.92 \mathrm{~A}$ & $38.92 \mathrm{~A}$ & $38.92 \mathrm{~A}$ & $32.33 \mathrm{D}-\mathrm{N}$ & $31.83 \mathrm{E}-\mathrm{O}$ & $32.08 \mathrm{H}-\mathrm{L}$ & $35.50 \mathrm{~A}$ \\
\hline Yearxvarieties & $32.84 \mathrm{~A}$ & $32.83 \mathrm{~A}$ & & $27.48 \mathrm{C}$ & $31.02 \mathrm{~B}$ & & \\
\hline Year & & & & & & & \\
\hline
\end{tabular}

Table 2 shows the lipid contents of seeds. The highest value was obtained from SP $245+\mathrm{OG}$ (45.90\%), CR (44.48\%) and SIP+OG (44.26\%) whereas the lowest value was observed from applications CONV. (37.24\%) and SP 245 (39.41\%). 
Table 2. Content of Lipid Obtained from Nusem and Beppo Seeds of Different Plant Activators and Organic Fertilizer Applications (\%)

\begin{tabular}{|c|c|c|c|c|c|c|c|}
\hline \multirow[b]{2}{*}{ Applications } & \multicolumn{2}{|c|}{2015} & \multirow{2}{*}{$\begin{array}{c}\text { Applications } \\
\text { x year }\end{array}$} & \multicolumn{2}{|c|}{2016} & \multirow{2}{*}{$\begin{array}{l}\text { Applications } \\
\text { x year }\end{array}$} & \multirow{2}{*}{$\begin{array}{l}\text { Applications } \\
\text { Average }\end{array}$} \\
\hline & Nusem & Beppo & & Nusem & Beppo & & \\
\hline $\mathrm{CR}$ & $54.00 \mathrm{~A}$ & $42.54 \mathrm{M}-\mathrm{b}$ & $48.27 \mathrm{~A}$ & $43.44 \mathrm{~K}-\mathrm{W}$ & 37.96 X-e & $40.70 \mathrm{~K}-\mathrm{O}$ & $44.48 \mathrm{AB}$ \\
\hline EM1 & $46.37 \mathrm{D}-\mathrm{O}$ & $39.92 \mathrm{~S}-\mathrm{b}$ & 43.15 B-N & $45.04 \mathrm{H}-\mathrm{S}$ & $37.56 \mathrm{Z}-\mathrm{f}$ & $41.30 \mathrm{H}-\mathrm{N}$ & $42.22 \mathrm{~B}-\mathrm{F}$ \\
\hline ERS & 49.84 A-I & $40.80 \mathrm{P}-f$ & $45.32 \mathrm{~A}-\mathrm{E}$ & $48.20 \mathrm{~B}$ & $32.46 \mathrm{fgh}$ & 40.33 L-P & 42.83 B-E \\
\hline VIT & $46.30 \mathrm{D}-\mathrm{O}$ & $37.24 b-f$ & $41.77 \mathrm{~F}-\mathrm{N}$ & $43.27 \mathrm{~K}-\mathrm{X}$ & $36.22 \mathrm{e}-\mathrm{f}$ & $39.74 \mathrm{~N}-\mathrm{Q}$ & $40.76 \mathrm{E}-\mathrm{H}$ \\
\hline OSU & $45.25 \mathrm{H}-\mathrm{S}$ & $36.72 \mathrm{e}-\mathrm{f}$ & $40.99 \mathrm{I}-\mathrm{N}$ & $44.83 \mathrm{I}-\mathrm{T}$ & $38.56 \mathrm{~W}-\mathrm{e}$ & $41.69 \mathrm{~F}-\mathrm{N}$ & $41.34 \mathrm{D}-\mathrm{H}$ \\
\hline M3 & $43.98 \mathrm{~J}-\mathrm{V}$ & $41.72 \mathrm{~N}-\mathrm{e}$ & 42.85 B-N & $42.51 \mathrm{M}-\mathrm{b}$ & $38.50 \mathrm{~W}-\mathrm{e}$ & 40.50 L-P & $41.68 \mathrm{C}-\mathrm{H}$ \\
\hline SP 245 & 47.09 C-M & $40.22 \mathrm{Q}-\mathrm{a}$ & 43.66 B-L & 40.77 P-f & $29.56 \mathrm{~h}$ & $35.17 \mathrm{R}$ & $39.41 \mathrm{HI}$ \\
\hline SIP & $45.53 \mathrm{G}-\mathrm{Q}$ & $38.49 \mathrm{~W}-\mathrm{e}$ & $42.01 \mathrm{D}-\mathrm{N}$ & $46.74 \mathrm{D}-\mathrm{N}$ & $36.32 \mathrm{~d}-\mathrm{f}$ & $41.53 \mathrm{G}-\mathrm{N}$ & $41.77 \mathrm{C}-\mathrm{G}$ \\
\hline EKO & $46.61 \mathrm{D}-\mathrm{N}$ & $41.70 \mathrm{~N}-\mathrm{\epsilon}$ & $44.15 \mathrm{~B}-\mathrm{K}$ & $45.63 \mathrm{G}-\mathrm{P}$ & $41.68 \mathrm{~N}-\mathrm{d}$ & 43.66 B-L & $43.91 \mathrm{ABC}$ \\
\hline BOT & $51.06 \mathrm{~A}-\mathrm{E}$ & $38.73 \mathrm{~V}-\mathrm{e}$ & $44.90 \mathrm{~A}-\mathrm{G}$ & $49.25 \mathrm{~A}-\mathrm{J}$ & $35.28 \mathrm{a}-\mathrm{g}$ & $42.26 \mathrm{C}-\mathrm{N}$ & $43.58 \mathrm{BCD}$ \\
\hline ZIN & 48.88 B-J & $39.88 \mathrm{~S}-\mathrm{b}$ & 44.38 B-J & $47.37 \mathrm{~B}-\mathrm{M}$ & 33.54 e-h & $40.45 \mathrm{~L}-\mathrm{P}$ & 42.42 B-E \\
\hline $\mathrm{CR}+\mathrm{OG}$ & $50.73 \mathrm{~A}-\mathrm{G}$ & 39.99 R-b & $45.36 \mathrm{~A}-\mathrm{D}$ & 45.31 H-R & $38.32 \mathrm{~W}-\mathrm{e}$ & $41.81 \mathrm{E}-\mathrm{N}$ & $43.59 \mathrm{BCD}$ \\
\hline EM1+OG & $52.47 \mathrm{AB}$ & 37.85 Z-e & $45.16 \mathrm{~A}-\mathrm{F}$ & $48.08 \mathrm{~B}-\mathrm{K}$ & $34.20 \mathrm{~d}-\mathrm{h}$ & $41.14 \mathrm{H}-\mathrm{N}$ & $43.15 \mathrm{BCD}$ \\
\hline ERS+OG & 46.04 E-P & $38.66 \mathrm{~V}-\mathrm{e}$ & $42.35 \mathrm{C}-\mathrm{N}$ & $48.03 \mathrm{~B}-\mathrm{K}$ & $34.64 \mathrm{~b}-\mathrm{h}$ & $41.33 \mathrm{H}-\mathrm{N}$ & $41.84 \mathrm{C}-\mathrm{G}$ \\
\hline $\mathrm{VIT}+\mathrm{OG}$ & 47.91 B-L & $43.24 \mathrm{~K}-\mathrm{Y}$ & $45.58 \mathrm{ABC}$ & 47.82 B-M & 35.58 e-f & $41.70 \mathrm{~F}-\mathrm{N}$ & $43.64 \mathrm{ABC}$ \\
\hline $\mathrm{OSU}+\mathrm{OG}$ & $46.33 \mathrm{D}-\mathrm{O}$ & $40.75 \mathrm{P}-\mathrm{f}$ & $43.54 \mathrm{~B}-\mathrm{L}$ & $38.98 \mathrm{~V}-\mathrm{d}$ & $34.49 \mathrm{c}-\mathrm{h}$ & $36.74 \mathrm{QR}$ & $40.14 \mathrm{FGH}$ \\
\hline $\mathrm{M} 3+\mathrm{OG}$ & $46.22 \mathrm{D}-\mathrm{O}$ & 42.62 L--a & 44.42 B-I & $46.66 \mathrm{D}-\mathrm{N}$ & $38.26 \mathrm{~W}-\mathrm{e}$ & $42.46 \mathrm{C}-\mathrm{N}$ & $43.44 \mathrm{BCD}$ \\
\hline SP 245+OG & $50.84 \mathrm{~A}-\mathrm{G}$ & $41.51 \mathrm{~N}-\mathrm{d}$ & $46.18 \mathrm{AB}$ & $52.18 \mathrm{ABC}$ & $39.06 \mathrm{~V}-\mathrm{d}$ & $45.62 \mathrm{ABC}$ & $45.90 \mathrm{~A}$ \\
\hline $\mathrm{SIP}+\mathrm{OG}$ & $50.24 \mathrm{~A}-\mathrm{H}$ & $39.63 \mathrm{~T}-\mathrm{c}$ & $44.93 \mathrm{~A}-\mathrm{G}$ & 45.94 E-P & $41.22 \mathrm{~N}-\mathrm{e}$ & 43.58 B-L & $44.26 \mathrm{AB}$ \\
\hline OSU+M3 & 45.67 F-P & $38.29 \mathrm{~W}-\mathrm{e}$ & $41.98 \mathrm{D}-\mathrm{N}$ & $37.90 \mathrm{Y}-\mathrm{e}$ & $36.61 e-f$ & $37.25 \mathrm{O}-\mathrm{R}$ & $39.61 \mathrm{GH}$ \\
\hline OSU+SP 245 & $51.51 \mathrm{~A}-\mathrm{D}$ & $37.52 \mathrm{a}-\mathrm{f}$ & $44.51 \mathrm{~B}-\mathrm{H}$ & 48.22 B-K & $38.42 \mathrm{~W}-\mathrm{e}$ & 43.32 B-M & $43.92 \mathrm{ABC}$ \\
\hline M3+SP 245 & $44.52 \mathrm{I}-\mathrm{Y}$ & $41.43 \mathrm{~N}-\mathrm{e}$ & 42.97 B-N & $43.39 \mathrm{~K}-\mathrm{W}$ & $30.62 \mathrm{gh}$ & $37.00 \mathrm{PQR}$ & $39.99 \mathrm{FGH}$ \\
\hline OG & $51.02 \mathrm{~A}-\mathrm{F}$ & $39.29 \mathrm{U}-\mathrm{d}$ & $45.15 \mathrm{~A}-\mathrm{F}$ & $42.90 \mathrm{~K}-\mathrm{Z}$ & $38.84 \mathrm{~V}-\mathrm{d}$ & $40.87 \mathrm{~J}-\mathrm{N}$ & 43.01 B-E \\
\hline CONV. & $41.70 \mathrm{~N}-\mathrm{e}$ & $38.01 \mathrm{X}-\mathrm{e}$ & $39.85 \mathrm{MQ}$ & $38.52 \mathrm{~W}-\mathrm{e}$ & $30.73 \mathrm{gh}$ & $34.62 \mathrm{R}$ & $37.24 \mathrm{I}$ \\
\hline Yearxvarieties & $47.92 \mathrm{~A}$ & $39.19 \mathrm{C}$ & & $45.04 \mathrm{~B}$ & $36.19 \mathrm{D}$ & & \\
\hline Year & & $\mathrm{A}$ & & & & & \\
\hline
\end{tabular}

Table 3 shows dominant fatty acids found in oils of seeds. Total 11 fatty acids were identified. Among them, the highest proportions in oil of snack seed pumpkin were C16:0 (Palmitic Acid Methyl Ester), C18:0 (Stearic Acid Methyl Ester), C18:1 (Oleic Acid Methyl Ester) and C18:2 (Linoleic Acid Methyl Ester).

Ermis [8] reported that the pumpkin seeds contained high lipid (35\%-48\%), and also oleic acid (40\%-58\%) and linoleic acid (30\%-40\%) were dominant fatty acids. He also reported that it contained $35 \%-40 \%$ of protein. These results were similar to the results of our study. However, the values were found to be different for oleic acid, linoleic acid and protein. Stevenson et al. [9] reported that the oil content of pumpkin cultivars ranged from 10.9 to $30.9 \%$. Researchers also reported the predominant fatty acids in pumpkin seed oil from all 12 cultivars studied as linoleic acid, oleic acid, palmitic acid, and stearic acid. These results were similar to findings of our study but values for oil content were found to be different. Lazoz [10] investigated the nutritional, fatty acid, and oil characteristics of pumpkin and melon seeds. As a result, they found the defatted flour of the pumpkin and melon seeds contained, respectively: crude protein, 55.4 and $39.4 \%$; and the major fatty acid of the oils was linoleic (18:2) at concentrations of 43.1 and $64.6 \%$, followed by oleic (18:1), 37.8 and 20.1\%, respectively. Habib et al [11] reported that the seeds of pumpkin (Curcurbita maxima) contained 36.70\% total lipid, 34.56\% total protein and pumpkin seed oil contained the highest amount of oleic acid (40.58\%) while stearic acid, palmitic acid and linoleic acid content were found to be $27.06,17.39$ and $14.97 \%$. These result were similar to the results of our study but values for protein, lipid, palmitic and linoleic acid were found to be different. 
Table 3. Content of dominant fatty acids; C16:0, C18:0, C18:1n9 and C18:2.

\begin{tabular}{|c|c|c|c|c|c|c|c|c|}
\hline \multirow[b]{2}{*}{ Applications } & \multicolumn{4}{|c|}{2015} & \multicolumn{4}{|c|}{2016} \\
\hline & C16:0 & C18:0 & C18:1n9 & C18:2 & C16:0 & C18:0 & C18:1n9 & C18:2 \\
\hline $\mathrm{CR}$ & $11.03 \mathrm{I}-\mathrm{N}$ & $6.16 \mathrm{~L}-\mathrm{R}$ & $42.03 \mathrm{~W}-\mathrm{a}$ & $38.12 \mathrm{CDE}$ & 11.57 C-M & $7.45 \mathrm{~A}-\mathrm{G}$ & $46.55 \mathrm{C}-\mathrm{D}$ & $31.19 \mathrm{VW}$ \\
\hline EM1 & 11.79 A-K & $6.53 \mathrm{G}-\mathrm{Q}$ & $41.11 \mathrm{Zab}$ & $38.23 \mathrm{CDE}$ & $11.79 \mathrm{~A}-\mathrm{K}$ & 7.10 B-L & $44.57 \mathrm{H}-\mathrm{L}$ & $33.57 \mathrm{~L}-\mathrm{Q}$ \\
\hline ERS & $11.53 \mathrm{C}-\mathrm{N}$ & $6.20 \mathrm{~L}-\mathrm{R}$ & $43.88 \mathrm{LQ}$ & $35.95 \mathrm{HI}$ & 12.40 A-D & 7.39 A-H & $44.96 \mathrm{G}-\mathrm{K}$ & $32.30 \mathrm{STU}$ \\
\hline VIT & 11.66 B-M & $6.51 \mathrm{G}-\mathrm{Q}$ & $42.75 \mathrm{RX}$ & $36.64 \mathrm{GH}$ & $12.22 \mathrm{~A}-\mathrm{G}$ & 7.72 A-D & $44.46 \mathrm{I}-\mathrm{N}$ & $32.39 \mathrm{R}-\mathrm{U}$ \\
\hline OSU & $11.32 \mathrm{D}-\mathrm{N}$ & $6.51 \mathrm{G}-\mathrm{Q}$ & $42.94 \mathrm{QW}$ & $36.61 \mathrm{GH}$ & $11.26 \mathrm{E}-\mathrm{N}$ & $7.95 \mathrm{AB}$ & $45.47 \mathrm{E}-\mathrm{I}$ & $32.86 \mathrm{O}-\mathrm{T}$ \\
\hline M3 & $11.72 \mathrm{~A}-\mathrm{L}$ & 6.68 E-P & $42.24 \mathrm{U}-\mathrm{Y}$ & $36.97 \mathrm{FGH}$ & $11.52 \mathrm{C}-\mathrm{N}$ & $7.61 \mathrm{~A}-\mathrm{E}$ & $47.25 \mathrm{BC}$ & $30.49 \mathrm{WX}$ \\
\hline SP 245 & $11.70 \mathrm{~A}-\mathrm{L}$ & $6.16 \mathrm{~L}-\mathrm{R}$ & $41.06 \mathrm{ab}$ & $38.57 \mathrm{BCD}$ & $12.68 \mathrm{AB}$ & 7.75 A-D & $42.58 \mathrm{~T}-\mathrm{X}$ & $33.40 \mathrm{M}-\mathrm{R}$ \\
\hline SIP & $10.95 \mathrm{~J}-\mathrm{N}$ & 6.69 E-P & $46.35 \mathrm{C}-\mathrm{F}$ & $33.75 \mathrm{KP}$ & $11.92 \mathrm{~A}-\mathrm{K}$ & $7.63 \mathrm{~A}-\mathrm{E}$ & $43.18 \mathrm{P}-\mathrm{V}$ & $33.92 \mathrm{~K}-\mathrm{N}$ \\
\hline EKO & $10.46 \mathrm{~N}$ & $6.54 \mathrm{~F}-\mathrm{Q}$ & $41.85 \mathrm{X}-\mathrm{a}$ & $38.67 \mathrm{ABC}$ & $11.88 \mathrm{~A}-\mathrm{K}$ & 6.70 E-P & $45.84 \mathrm{D}-\mathrm{G}$ & $32.69 \mathrm{P}-\mathrm{T}$ \\
\hline$\overline{\mathrm{BOT}}$ & $11.26 \mathrm{E}-\mathrm{N}$ & $6.41 \mathrm{I}-\mathrm{Q}$ & $41.33 \mathrm{YZa}$ & $36.16 \mathrm{HI}$ & $12.08 \mathrm{~A}-\mathrm{I}$ & $7.19 \mathrm{~B}-\mathrm{K}$ & $42.07 \mathrm{~W}-\mathrm{a}$ & $35.29 \mathrm{IJ}$ \\
\hline ZIN & $11.85 \mathrm{~A}-\mathrm{K}$ & $6.16 \mathrm{~L}-\mathrm{R}$ & $40.15 \mathrm{~b}$ & $39.29 \mathrm{AB}$ & $12.54 \mathrm{ABC}$ & $8.20 \mathrm{~A}$ & $44.36 \mathrm{~J}-\mathrm{N}$ & $31.62 \mathrm{UV}$ \\
\hline $\mathrm{CR}+\mathrm{OG}$ & $11.00 \mathrm{I}-\mathrm{N}$ & $6.11 \mathrm{~N}-\mathrm{R}$ & $44.50 \mathrm{I}-\mathrm{M}$ & $36.15 \mathrm{HI}$ & 11.79 A-K & 7.01 B-M & $45.58 \mathrm{D}-\mathrm{H}$ & $32.80 \mathrm{O}-\mathrm{T}$ \\
\hline EM1+OG & $11.20 \mathrm{~F}-\mathrm{N}$ & $5.69 \mathrm{QR}$ & $42.31 \mathrm{U}-\mathrm{Y}$ & $38.28 \mathrm{BE}$ & $12.34 \mathrm{~A}-\mathrm{E}$ & $7.50 \mathrm{~A}-\mathrm{F}$ & $45.84 \mathrm{D}-\mathrm{G}$ & $30.84 \mathrm{VWX}$ \\
\hline ERS+OG & $12.77 \mathrm{~A}$ & $5.86 \mathrm{O}-\mathrm{R}$ & $45.63 \mathrm{D}-\mathrm{G}$ & $35.45 \mathrm{IJ}$ & $12.26 \mathrm{~A}-\mathrm{F}$ & $7.80 \mathrm{ABC}$ & $43.41 \mathrm{~N}-\mathrm{T}$ & $33.34 \mathrm{~N}-\mathrm{S}$ \\
\hline $\mathrm{VIT}+\mathrm{OG}$ & $11.10 \mathrm{H}-\mathrm{N}$ & $6.05 \mathrm{M}-\mathrm{R}$ & $44.27 \mathrm{~K}-\mathrm{O}$ & $36.18 \mathrm{HI}$ & $12.40 \mathrm{~A}-\mathrm{D}$ & $6.74 \mathrm{E}-\mathrm{O}$ & 45.44 E-I & $32.52 \mathrm{Q}-\mathrm{U}$ \\
\hline OSU+OG & $10.57 \mathrm{MN}$ & $6.56 \mathrm{~F}-\mathrm{Q}$ & $43.41 \mathrm{M}-\mathrm{T}$ & $36.91 \mathrm{FGH}$ & $12.00 \mathrm{~A}-\mathrm{J}$ & $6.80 \mathrm{D}-\mathrm{O}$ & $43.65 \mathrm{~L}-\mathrm{S}$ & $34.01 \mathrm{~K}-\mathrm{N}$ \\
\hline $\mathrm{M} 3+\mathrm{OG}$ & $11.32 \mathrm{D}-\mathrm{N}$ & $6.20 \mathrm{~L}-\mathrm{R}$ & $43.04 \mathrm{P}-\mathrm{W}$ & $37.26 \mathrm{EFG}$ & $11.52 \mathrm{C}-\mathrm{N}$ & 6.94 C-N & $46.50 \mathrm{CDE}$ & $32.25 \mathrm{TU}$ \\
\hline SP $245+O G$ & $11.28 \mathrm{E}-\mathrm{N}$ & $6.44 \mathrm{H}-\mathrm{Q}$ & $41.83 \mathrm{X}-\mathrm{a}$ & $37.94 \mathrm{CF}$ & $12.16 \mathrm{~A}-\mathrm{H}$ & $6.97 \mathrm{C}-\mathrm{N}$ & $44.55 \mathrm{H}-\mathrm{M}$ & $33.28 \mathrm{~N}-\mathrm{T}$ \\
\hline $\mathrm{SIP}+\mathrm{OG}$ & $10.84 \mathrm{~K}-\mathrm{N}$ & $5.27 \mathrm{R}$ & $44.09 \mathrm{~K}-\mathrm{P}$ & $36.61 \mathrm{GH}$ & 11.95 A-J & 7.35 A-I & $43.21 \mathrm{P}-\mathrm{U}$ & $34.50 \mathrm{JKL}$ \\
\hline OSU+M3 & 11.65 B-M & $6.24 \mathrm{~K}-\mathrm{Q}$ & $42.13 \mathrm{~V}-\mathrm{Z}$ & $37.56 \mathrm{DG}$ & 11.74 A-L & $7.23 \mathrm{~B}-\mathrm{J}$ & $43.50 \mathrm{M}-\mathrm{T}$ & $34.45 \mathrm{~J}-\mathrm{M}$ \\
\hline OSU+SP 245 & $11.22 \mathrm{~F}-\mathrm{N}$ & 5.76 PQR & 43.74 L-R & $36.82 \mathrm{GH}$ & $11.13 \mathrm{G}-\mathrm{N}$ & $6.81 \mathrm{D}-\mathrm{O}$ & 45.40 F-J & $33.75 \mathrm{~K}-\mathrm{O}$ \\
\hline M3+SP 245 & 11.58 B-M & $6.36 \mathrm{~J}-\mathrm{Q}$ & 42.94 Q-W & $36.52 \mathrm{GH}$ & $12.36 \mathrm{~A}-\mathrm{E}$ & $6.86 \mathrm{C}-\mathrm{N}$ & $43.24 \mathrm{O}-\mathrm{U}$ & $34.60 \mathrm{JKL}$ \\
\hline OG & $10.65 \mathrm{LMN}$ & 6.18 L-R & $41.26 \mathrm{YZa}$ & $39.63 \mathrm{~A}$ & $12.59 \mathrm{ABC}$ & 7.02 B-M & $42.62 \mathrm{~S}-\mathrm{X}$ & $34.80 \mathrm{JK}$ \\
\hline CONV. & $11.31 \mathrm{D}-\mathrm{N}$ & $6.64 \mathrm{~F}-\mathrm{Q}$ & $48.34 \mathrm{~A}$ & 31.53 UVW & 11.59 B-M & 7.75 A-D & $47.80 \mathrm{AB}$ & $29.87 \mathrm{X}$ \\
\hline
\end{tabular}

\section{Conclusions}

In our study eight plant activators and three organic fertilizer were used in snack seed pumpkin with or without organic fertilizers. Total protein ranged from $28.32 \%$ to $35.50 \%$, and total lipid ranged from $37.24 \%$ to $45.90 \% .11$ fatty acid were identified. C16:0, C18:0, C18:1n9 and C18:2 were found dominant compared to other fatty acids. As a result, applied plant activators on Nusem and Beppo varieties had no effect total protein content, total lipid and fatty acid in snack seed pumpkin.

\section{Conflict of Interest}

The authors declare that there is no conflict of interest.

\section{Acknowledgements}

This project was funded by Cukurova University Scientific Research Projects Unit with the project code of FDK-2015-3664. The researcher thanks to Prof. Dr. Fikrettin Sahin from Yeditepe University, Turkey for providing isolates which are OSU 142, SP 245 and M3.

\section{References}

[1] TUIK, http://www.tuik.gov.tr, 2017.

[2] G. Meru et al., Health benefits of pumpkin seed and nutrition profile of 35 pumpkin accessions, UF/IFAS Extension, Gainesville, 2017, FL 3261.

[3] A. Günes, organic and conventional fertilization snack seed pumpkin in growth, in: Snack Seed Workshop, 26-27 November 2014, pp. 81-87. 
[4] AOAC. Official Method 955.04, Nitrogen (Total) in Seafood. Hungerford JM, chapter editor. In: Cunniff, editor. Fish and Other Marine Products. Official Methods of Analysis of AOAC International, USA, 1998, Chapter 35, p. 6.

[5] E.G. Bligh, W.J. Dyer, A rapid method of total lipid extraction and purification, Biochemistry and Cell Biology. 37(8) (1959) 911917.

[6] M. Kenar, Investigation of sensory, chemical and microbiological effects of natural antioxidant obtained from aromatic plants on fish fillets, MSc. Thesis, Department of Fisheries, University of Cukurova, Adana, Turkey, 2009, Pages: 80.

[7] I. Ken'ichi et al., An improved method for rapid analysis of the fatty acids of glycerolipids, Lipids. 31(5) (1996) 535-539.

[8] S. Ermis, The effect of ecology on seed production and snack quality of pumpkin (Cucurbita pepo L.) in Turkey, PhD. Thesis, Department of Horticulture, Ankara Universirty, Ankara, Turkey, 2010, Pages: 153.

[9] D.G. Stevenson et al., Oil and tocopherol content and composition of pumpkin seed oil in 12 cultivars, Food Science and Human Nutrition. 55(10) (2007) 4005-4013.

[10] E.S. Lazos, Nutritional, fatty acid, and oil characteristics of pumpkin and melon seeds, Journal of Food Science. 51(5) (1986) 1365-2621.

[11] A. Habib et al., Nutritional and lipid composition analysis of pumpkin seed (Cucurbita maxima linn.), Journal of Nutrition \& Food Sciences. 5(4) (2015). 